\title{
Risk of Overdose with Exposure to Prescription Opioids, Benzodiazepines, and Non-benzodiazepine Sedative-Hypnotics in Adults: a Retrospective Cohort Study
}

\author{
Joanne Cho, PharmD, $\mathrm{BCPS}^{7}$, Michele M. Spence, $\mathrm{PhD}{ }^{2}$, Fang Niu, $\mathrm{MS}^{2}$, \\ Rita L. Hui, PharmD, MS ${ }^{2,3}$, Patricia Gray, PharmD ${ }^{4}$, and Steven Steinberg, $M D^{5}$
}

${ }^{1}$ Pharmacy Services , Kaiser Permanente, Bakersfield, CA, USA; ${ }^{2}$ Pharmacy Outcomes Research Group, Downey, CA, USA; ${ }^{3}$ Pharmacy Outcomes Research Group, Oakland, CA, USA; ${ }^{4}$ Pharmacy Services, Kaiser Permanente, Riverside, CA, USA; ${ }^{5}$ Southern California Permanente Medical Group, Pasadena, USA.

BACKGROUND: Concurrent use of benzodiazepines in opioid users has been linked to a higher risk of an emergency room visit or inpatient admission for opioid overdose and death from drug overdose. Further research is needed to confirm the findings and analyze contributing risk factors for opioid overdoses in a large commercially insured population.

OBJECTIVES: To estimate the risk of opioid overdose associated with opioid users exposed to various combinations of opioid, benzodiazepine, and non-benzodiazepine sedative-hypnotic therapy. To identify other factors that are associated with increased risk for opioid overdose.

DESIGN: Retrospective cohort study.

PATIENTS: New start adult users of opioids, defined as naïve to opioids for 6 months, in Kaiser Permanente California regions from January 2013 through September 2017.

MAIN MEASURES: Inpatient or emergency department admissions due to opioid-related overdose.

KEY RESULTS: A total of 2,241,530 patients were included in this study. Patients exposed to opioids, benzodiazepines, and non-benzodiazepine sedative-hypnotics at any point during their follow-up were $60 \%$ more likely to overdose than those who were only exposed to opioids ( $p<$ 0.0001). Those exposed to opioids and benzodiazepines were $20 \%$ more likely to have an opioid-related overdose than those exposed to opioids only $(p<0.0001)$. Significant risk factors for opioid overdose included exposure to all three medication classes, higher opioid dosage strengths, elderly age (age $\geq 65$ years), history of previous overdose, and substance use disorder.

CONCLUSIONS: Results from this study demonstrate a significant increase in risk of opioid overdose in patients exposed to combinations of sedative-hypnotics with opioids compared to those only taking opioids. Findings from

Prior Presentations This research was presented as a poster at the Academy of Managed Care Pharmacy Annual Meeting in Boston, MA, in 2018 and as a presentation at the Western States Conference in San Diego, CA, in 2018 and California Society of Health-System Pharmacists Seminar in San Diego, CA, in 2018.

Electronic supplementary material The online version of this article (https://doi.org/10.1007/s11606-019-05545-y) contains supplementary material, which is available to authorized users.

Received February 21, 2019

Revised August 2, 2019

Accepted October 10, 2019

Published online January 9, 2020 this study provide evidence that opioids should be avoided in combination with benzodiazepines and nonbenzodiazepine sedative-hypnotics, used at the lowest dose possible, and used with caution in the elderly, those with previous history of overdose, and those with substance use disorder at baseline.

KEY WORDS: patient centered outcomes research; medical safety; risk assessment; pain.

J Gen Intern Med 35(3):696-703 DOI: $10.1007 / \mathrm{s} 11606-019-05545-\mathrm{y}$

(C) Society of General Internal Medicine 2020

\section{INTRODUCTION}

Opioid overdose has been declared as a national health epidemic by the Centers for Disease Control and Prevention $(\mathrm{CDC})^{1}$ and over 1,000 people are treated in emergency departments daily due to misuse of prescription opioids ${ }^{2}$.

Benzodiazepines and other sedative-hypnotics can increase overdose risk by exacerbating opioid-induced respiratory depression and potentiating central nervous system depression. According to the National Institute on Drug Abuse, more than 30\% of opioid-related overdoses involve benzodiazepines ${ }^{3}$. Concurrent use of benzodiazepines in opioid users has been linked to a higher risk of an emergency room visit or inpatient admission for opioid overdose $^{4}$ and death from drug overdose ${ }^{5}$. Previous research has shown that concurrent use of prescription opioids and sedativehypnotics, even at low to moderate opioid doses, increases the opioid mortality risk in Medicaid patients ${ }^{6}$.

Although there is evidence regarding the impact of concurrent use of benzodiazepines and chronic opioid use on opioidrelated overdoses or deaths, other risk factors for opioid overdose in the commercially insured population are not fully understood.

Kaiser Permanente (KP) is a not-for-profit integrated health care delivery system that provides care to 12.3 million members across the USA, in 8 states plus the District of Columbia 7. Our fully integrated healthcare system allows us to have access to electronic prescribing data and detailed patient records that we have used to identify opportunities to drive 
patient care initiatives (i.e., opioid dose tapering and use of evidence-based non-opioid and non-drug pain management alternatives) in order to ensure appropriate and safe use of opioids for acute and chronic pain management.

Although KP has achieved success in improving appropriate opioid prescribing practice ${ }^{8}$, it is still crucial to understand the risks associated with opioid overdose and to quantify those risks. Thus, this study was conducted to estimate the risk of opioid overdose associated with opioid users receiving various combinations of opioid, benzodiazepine, and nonbenzodiazepine sedative-hypnotic therapy, and to identify factors that may be associated with an increased risk for opioid overdose.

\section{METHODS}

We report findings from a retrospective cohort database study conducted at KP. The study was approved by the Institutional Review Boards for KP Northern and Southern California.

\section{Study Population}

The cohort identification period of this study ran from January 1, 2013, to September 30, 2017, and included patients who started a new episode of opioid use (no opioid prescription filled in the past 6 months) and were at least 18 years of age with continuous medical and pharmacy benefits for at least 12 months before the index date. The first opioid prescription sold during the study period for each patient was defined as the index prescription date. Patients were excluded if they had a diagnosis of cancer within 6 months prior to index prescription or a diagnosis of cancer (except nonmelanoma skin cancer) during the follow-up period. Those who were in palliative care, hospice care, or skilled nursing facilities during the index date were also excluded. Follow-up began at the index prescription date and continued until whichever occurred first: the first opioid-related overdose, death, end of membership, diagnosis of cancer, or the end of study (December 31, 2017).

\section{Definition of Opioid-Related Overdose}

The primary outcome was an emergency department or hospital admission due to opioid-related overdose, which was defined using International Classification of Diseases, Ninth Revision (ICD-9) or Tenth Revision (ICD-10) codes (See Appendix online, Table 1). Only the first opioid-related overdose event during the follow-up period was considered. Subsequent overdose events were not evaluated. Prior opioidrelated overdose was also ascertained using the same definition for the 12 months prior to index date. Since the cohort included patients who were naïve to opioids for 6 months prior to the index date, it is possible that these patients may have been prescribed opioids within 7-12 months prior to the index date and had an overdose occur within 12 months prior to index date.
Sensitivity analysis no. 1 looked at crude rates of overdose during the drug exposure period and within 90 days after the end of the prescription. The time interval of drug exposure was defined starting from the day the prescription was filled and lasting until the number of days supplied in the prescription. For example, if a patient's days' supply of an opioid prescription overlapped with the date of the outcome event, but the days' supply of a benzodiazepine prescription ended before the outcome event, the patient was classified in the "O only" group (See Appendix online, Figure 1). Outcome events that occurred outside of this time interval were classified as "remote use." The overall rate of opioid-related overdoses per 10,000 person-years was calculated, and the crude rates were stratified by exposure groups.

\section{Opioid Prescription Use and Dosing}

The KP pharmacy database contains records for each medication dispensed with information on drug name, strength, quantity, day supply, and dispense date. All drugs that contain the following opioid analgesics were included: buprenorphine, butorphanol, codeine, fentanyl, hydrocodone, hydromorphone, levorphanol, meperidine, methadone, morphine, nalbuphine, opium tincture, oxycodone, oxymorphone, tapentadol, and tramadol. Methadone was included because methadone maintenance treatment for opioid addiction or detoxification is outsourced from KP. Therefore, outpatient prescriptions for methadone dispensed by KP are for pain management.

For each claim, the morphine milligram equivalent (MME) dose was calculated by using published conversion factors ${ }^{9}$. Then, the average daily MME was calculated for each patient in the cohort by dividing the sum of MMEs from all claims with the total day supply of opioids.

\section{Classification of Drug Exposure}

Benzodiazepines included in this study were alprazolam, chlordiazepoxide, clobazam, clonazepam, clorazepate, diazepam, estazolam, flurazepam, halazepam, lorazepam, midazolam, oxazepam, prazepam, quazepam, temazepam, and triazolam. Non-benzodiazepine sedative-hypnotics included eszopiclone, zaleplon, and zolpidem.

Two different treatment grouping approaches were used. The first approach grouped the cohort into four general exposure categories based on exposure to benzodiazepines, nonbenzodiazepine sedative-hypnotics, or both over the follow-up period $(\mathrm{O}$ only, $\mathrm{O}+\mathrm{B}, \mathrm{O}+\mathrm{S}, \mathrm{O}+\mathrm{B}+\mathrm{S})$. If a patient filled prescriptions for a benzodiazepine and non-benzodiazepine sedative-hypnotic, either concurrently or subsequently, at any point during their follow-up period, the patient was classified in the opioid + benzodiazepine + non-benzodiazepine sedative-hypnotic $(\mathrm{O}+\mathrm{B}+\mathrm{S})$ group (Fig. 1). This grouping did not necessarily indicate that the patient was taking all three medications simultaneously. Due to the as-needed nature of use of all three groups of medications, this grouping denoted that the patient was in possession of all three medications $(\mathrm{O}+$ 


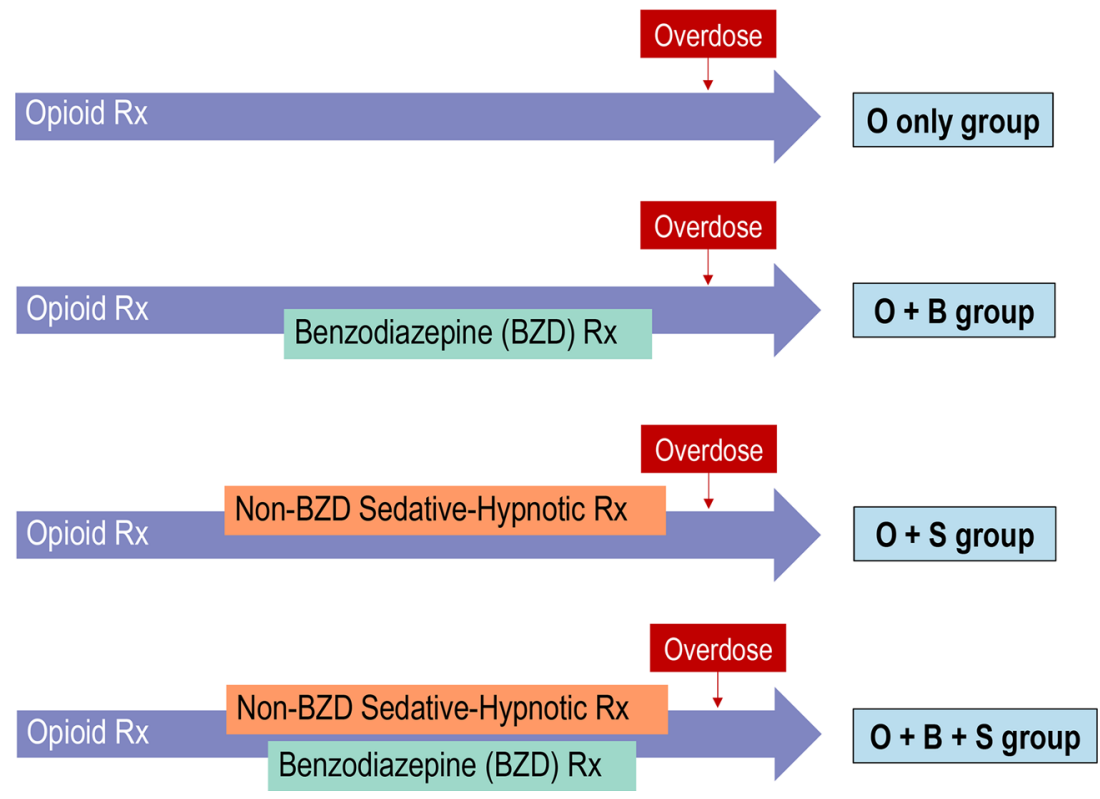

$\mathrm{O}=$ Opioid, $\mathrm{B}=$ Benzodiazepine, $\mathrm{S}=$ Non-benzodiazepine sedative-hypnotic, $\mathrm{Rx}=$ prescription

Figure 1 Drug exposure scenarios.

$\mathrm{B}+\mathrm{S}$ ) at any time during the follow-up period. In contrast, those in the "O only" group were only exposed to opioids and not to any sedative-hypnotics, while "O $+\mathrm{S}$ " and "O $+\mathrm{B}$ " implied that the patients received a non-benzodiazepine sedative-hypnotic prescription or benzodiazepine prescription on or after the initial opioid prescription. In sensitivity analysis no. 2, drug exposure was assigned as time-dependent variables based on the day supply of the prescription.

The second approach further stratified those four exposure groups into 10 groups based on the average daily opioid dose. Those exposed only to opioids were grouped into the following opioid dosage range groups: 1-20 MME/day, 21-49 $\mathrm{MME} /$ day, 50-89 MME/day, and $\geq 90 \mathrm{MME} /$ day. Those exposed to benzodiazepines and/or non-benzodiazepine sedative-hypnotics were grouped into 2 dosage range categories: 1-20 MME/day and > $20 \mathrm{MME} /$ day.

\section{Covariates}

Demographic and diagnostic data was extracted from electronic patient records. Demographic data included age, gender, race, and Medicaid status. Medical comorbidity was measured by utilizing the Charlson Comorbidity Index (CCI) score ${ }^{10}$ and assessed for the following comorbidities (diagnosed within 12 months prior to the index prescription) at baseline: depression, anxiety, other psychiatric disorders, substance use disorder, alcoholism, nicotine dependence, chronic fatigue syndrome, chronic pulmonary obstructive disease, asthma, pneumonia, and sleep apnea. Receipt of other drugs such as skeletal muscle relaxants (SMR), GABA-analogues, attention deficit hyperactivity disorder medications, and buprenorphine within 12 months of the index prescription was also assessed (see Appendix online, Table 2)

\section{Statistical Analysis}

For the univariate analysis, chi-square tests were used for categorical variables and Kruskal-Wallis tests for continuous variables. Two Cox proportional hazards models were utilized to assess associations between benzodiazepine and/or nonbenzodiazepine sedative-hypnotic use and risk of opioidrelated overdose. The first model was a regression analysis comparing 4 main exposure groups $(\mathrm{O}$ only, $\mathrm{O}+\mathrm{B}, \mathrm{O}+\mathrm{S}, \mathrm{O}+$ $\mathrm{B}+\mathrm{S})$ and the second regression model compared 10 groups, stratified from the 4 main groups based on average daily opioid MME categories. Both models were adjusted for demographics, depression, anxiety, other psychiatric disorders, substance use disorder, alcoholism, nicotine dependence, previous overdose, sleep apnea, SMR, GABA-analogues, buprenorphine, obesity, and weighted CCI. We presented the adjusted hazard ratios for these variables based on the first and second model in the results. For sensitivity analysis no. 2, a time-dependent Cox proportional hazards model was used. All $P$ values $<0.05$ are considered significant. All analyses were conducted using SAS 9.4 (SAS Institute Inc., Cary, NC).

\section{RESULTS}

A total of $3,022,622$ new start adult opioid users within the cohort identification period were identified and 539,583 were excluded due to lack of continuous membership and drug benefit. Of the remaining patients, 182,578 were excluded due to having a diagnosis of cancer. Subsequently, 58,391 were excluded due to hospice/palliative care or enrollment in a skilled nursing facility (Fig. 2). The final cohort consisted of $2,241,530$ patients and the median number of opioid 


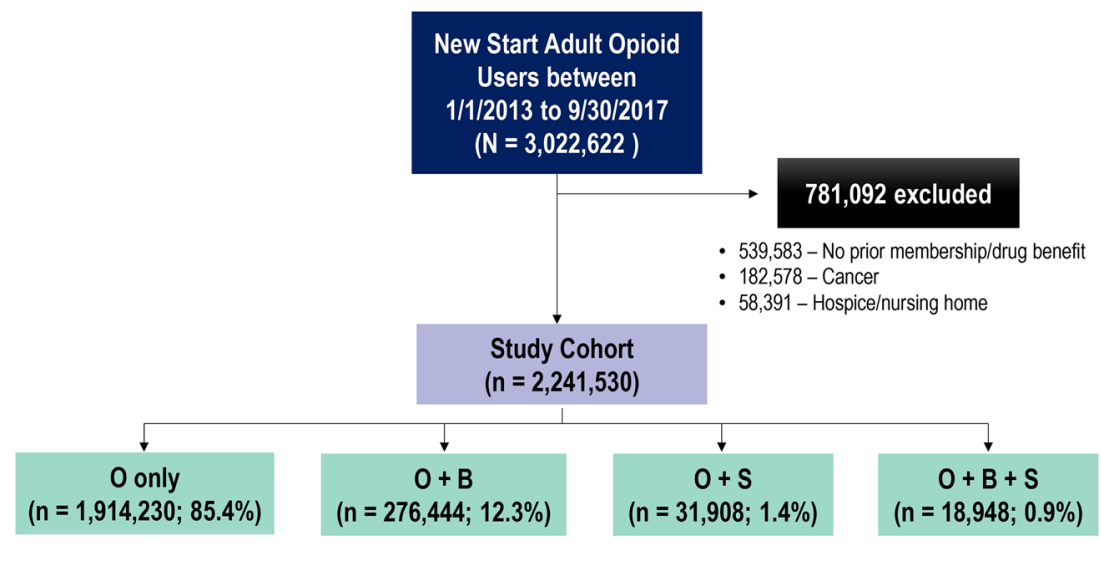

$\mathrm{O}=$ Opioid, $\mathrm{B}=$ Benzodiazepine, $\mathrm{S}=$ Non-benzodiazepine sedative-hypnotic

Figure 2 Cohort identification flowchart.

prescriptions per person was 2 (interquartile range [IQR] 1-3). Of these, $85.4 \%$ were exposed only to opioids, $12.3 \%$ to opioids and benzodiazepines, $1.4 \%$ to opioids and nonbenzodiazepine sedative-hypnotics, and $0.9 \%$ to all three medication classes (Fig. 2). The median follow-up time for the cohort was approximately 2.5 (IQR 1.3-3.9) years.

A total of 4,708 patients were admitted for an opioid-related overdose, which represented a crude rate of 8.2 per 10,000 person-years. Fifty-three percent of opioid overdoses occurred during the drug exposure period and $71 \%$ of opioid overdoses happened within 90 days after drug exposure.

\section{Baseline Characteristics}

Table 1 outlines the baseline characteristics of each exposure group. The mean age of the cohort ranged from 47.5 to 52.6 years. More than half the cohort $(43.7-64.4 \%)$ were of White race. A small percentage $(2.2-3.8 \%)$ were enrolled in Medicaid. In addition, 31-36\% of the cohort had a body mass index (BMI) $\geq 30$ or a diagnosis of obesity. Those exposed to all three medication classes had a higher percentage of depression and anxiety at baseline than those only exposed to opioids $(26.6 \%$ vs. $7.0 \%$ and $31.9 \%$ vs. $5.9 \%$, respectively, $p<0.0001)$.

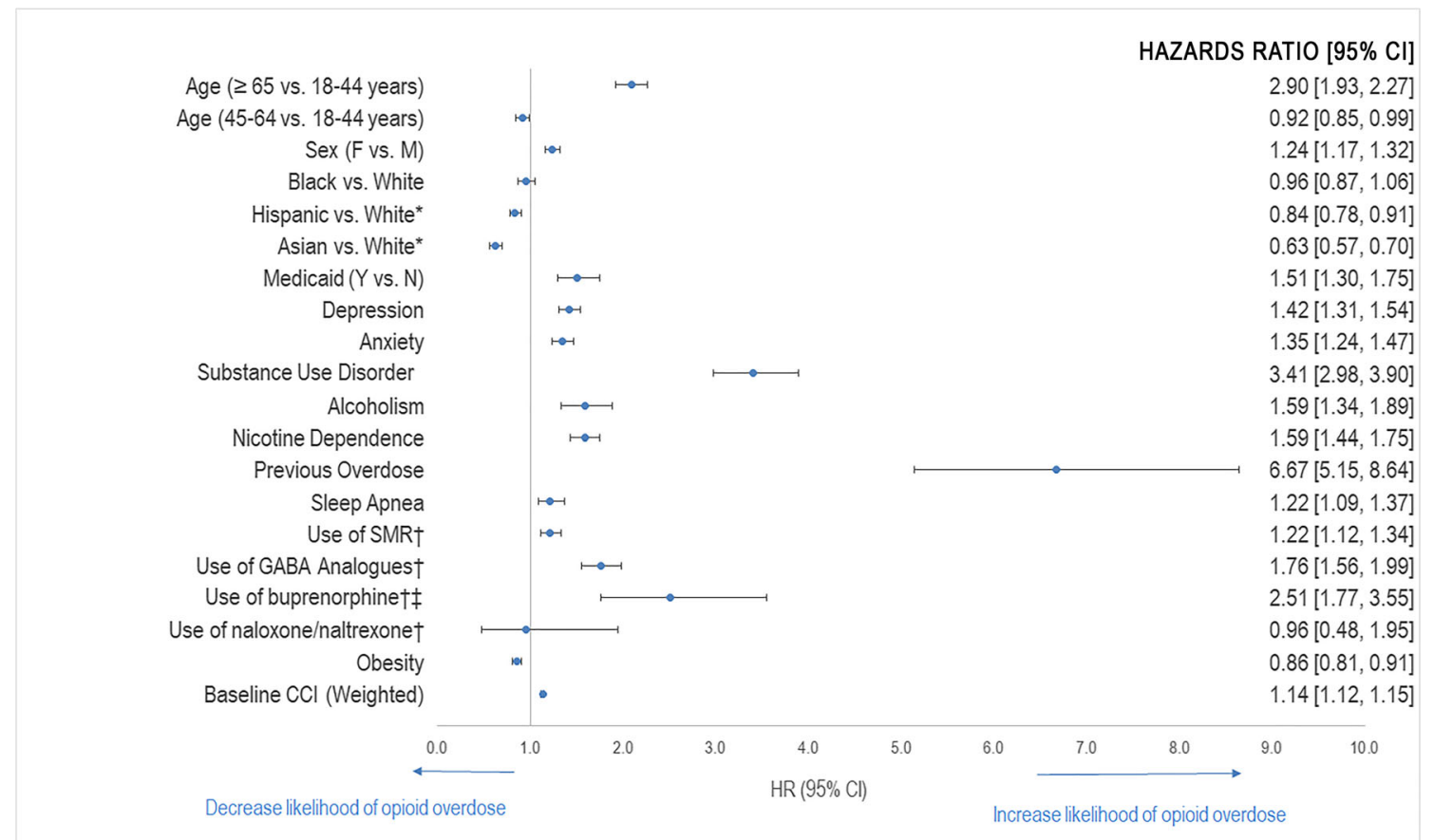

Figure 3 Factors associated with opioid-related overdose. Based on the regression model using drug exposure. *Not statistically significant. Defined as receipt of drug within 12 months of the index prescription date. *Includes buprenorphine/naloxone and buprenorphine for substance use disorder. 
Table 1 Baseline Characteristics

\begin{tabular}{|c|c|c|c|c|}
\hline Baseline characteristics & $\begin{array}{l}\text { O only } \\
(n=1,914,230)\end{array}$ & $\begin{array}{l}O+B \\
(n=276,444)\end{array}$ & $\begin{array}{l}\mathbf{O}+\mathbf{S} \\
(n=\mathbf{3 1}, 908)\end{array}$ & $\begin{array}{l}\mathrm{O}+\mathrm{B}+\mathrm{S} \\
(n=18,948)\end{array}$ \\
\hline Age, mean (SD) & $47.5(17.5)$ & $49.2(16.7)$ & $52.6(14.1)$ & $50.4(14.2)$ \\
\hline Female, $n(\%)$ & $1,037,124(54.2)$ & $195,733(70.8)$ & $17,870(56.0)$ & $12,934(68.3)$ \\
\hline \multicolumn{5}{|l|}{ Race, $n(\%)$} \\
\hline White & $836,291(43.7)$ & $150,281(54.4)$ & $20,005(62.7)$ & $12,207(64.4)$ \\
\hline African American & $179,021(9.3)$ & $23,874(8.6)$ & $2,160(6.8)$ & $1,322(7.0)$ \\
\hline Hispanic & $482,717(25.2)$ & $62,308(22.5)$ & $4,233(13.3)$ & $2,860(15.1)$ \\
\hline Asian & $280,671(14.7)$ & $24,032(8.7)$ & $3,818(11.9)$ & $1,531(8.1)$ \\
\hline Other/unknown & $135,530(7.1)$ & $15,949(5.8)$ & $1,692(5.3)$ & $1,028(5.4)$ \\
\hline Medicaid, $n(\%)$ & $68,331(3.6)$ & $10,573(3.8)$ & $704(2.2)$ & $579(3.1)$ \\
\hline BMI $\geq 30$ (obesity), $n(\%)$ & $679,531(35.5)$ & $100,851(36.5)$ & $9,908(31.1)$ & $5,991(31.6)$ \\
\hline $\mathrm{CCI}$ score, mean (SD) & $1.0(1.8)$ & $1.1(1.9)$ & $1.1(1.9)$ & $1.1(1.8)$ \\
\hline Previous overdose, $n(\%)$ & $933(0.05)$ & $296(0.11)$ & $42(0.13)$ & $29(0.15)$ \\
\hline Use of SMR, $n(\%)$ & $172,132(9.0)$ & $37,621(13.6)$ & $4,293(13.5)$ & $3,298(17.4)$ \\
\hline Use of GABA-analogues*, $n(\%)$ & $29,362(1.5)$ & $8,453(3.0)$ & $1,253(3.9)$ & $1,014(5.4)$ \\
\hline Use of ADHD meds*, $n(\%)$ & $14,209(0.7)$ & $4,150(1.5)$ & $784(2.5)$ & $651(3.4)$ \\
\hline Use of naloxone/naltrexone*, $n(\%)$ & $451(0.0)$ & $199(0.1)$ & $21(0.1)$ & $22(0.1)$ \\
\hline Use of buprenorphine ${ }^{* \dagger}, n(\%)$ & $243(0.0)$ & $124(0.0)$ & $6(0.0)$ & $16(0.1)$ \\
\hline \multicolumn{5}{|l|}{ Comorbidities, $n(\%)$} \\
\hline Depression & $133,121(7.0)$ & $48,685(17.6)$ & $5,376(16.9)$ & $5,040(26.6)$ \\
\hline Anxiety & $113,201(5.9)$ & $66,868(24.2)$ & $4,055(12.7)$ & $6,042(31.9)$ \\
\hline Other psychiatric disorders & $57,264(3.0)$ & $22,383(8.1)$ & $2,148(2.6)$ & $2,457(2.9)$ \\
\hline Substance use disorder & $19,027(1.0)$ & $4,977(1.8)$ & $468(1.5)$ & $534(2.8)$ \\
\hline Alcoholism & $10,424(0.5)$ & $3,690(1.3)$ & $343(1.1)$ & $337(1.8)$ \\
\hline Nicotine dependence & $101,417(5.3)$ & $18,724(6.8)$ & $1,656(5.2)$ & $1,269(6.7)$ \\
\hline Chronic fatigue syndrome & $615(0.03)$ & $238(0.09)$ & $45(0.14)$ & $29(0.15)$ \\
\hline COPD & $9,553(0.5)$ & $1,750(0.6)$ & $149(0.5)$ & $82(0.4)$ \\
\hline Asthma & $173,262(9.0)$ & 32,958 (11.9) & $3,547(11.1)$ & $2,433(12.8)$ \\
\hline Pneumonia & $11,087(0.6)$ & $1,126(0.4)$ & $118(0.4)$ & $71(0.6)$ \\
\hline Sleep apnea & $74,870(3.9)$ & $13,007(4.7)$ & $2,319(2.5)$ & $1,291(1.4)$ \\
\hline
\end{tabular}

$p<0.0001$ for all characteristics

$O=$ opioid, $B=$ benzodiazepine, $S=$ non-benzodiazepine sedative-hypnotic, $S D=$ standard deviation; $S M R=$ skeletal muscle relaxants, $C O P D=$ chronic obstructive pulmonary disease, $B M I=$ body mass index, CCI = Charlson Comorbidity Index, ADHD = attention deficit and hyperactivity disorder, GABA = gamma-aminobutyric acid

*Defined as receipt of drug within 12 months of the index prescription date

${ }^{\dagger}$ Includes buprenorphine/naloxone and buprenorphine for substance use disorder

\section{Risk of Overdose Based on Drug Exposure}

Overall, patients who were exposed to opioids, benzodiazepines, and non-benzodiazepine sedative-hypnotics $(\mathrm{O}+\mathrm{B}+\mathrm{S}$ group) had the highest risk of opioid-related overdose and were $60 \%$ more likely than those who were only exposed to opioids. Those exposed to opioids and benzodiazepines $(\mathrm{O}+$ B group) were $20 \%$ more likely than those only exposed to opioids. The results of the sensitivity analysis from the timedependent model were consistent with results from the main analysis(Table 2).
Across all exposure groups, we observed an increase in overdose risk with higher opioid doses. Compared to those who were only exposed to an opioid at doses $\leq 20 \mathrm{MME}$, patients exposed to all three medication classes and opioids at doses $>20 \mathrm{MME}$ were over four times more likely to overdose (Table 3 ).

\section{Factors Associated with Opioid-Related Overdose}

Hispanics and Asians were $20 \%$ and $40 \%$ less likely than Whites to be admitted for an opioid-related overdose, respectively.

Table 2 Risk of Overdose Based on Drug Exposure

\begin{tabular}{|c|c|c|c|}
\hline$\overline{\text { Exposure groups }}$ & $\begin{array}{l}\text { Unadjusted HR } \\
(95 \% \mathrm{CI}, p \text { value) } \\
N=\mathbf{2 , 2 4 1 , 5 3 0}\end{array}$ & $\begin{array}{l}\text { Adjusted HR } \\
\text { (95\% CI, } p \text { value) } \\
N=\mathbf{2 , 2 4 1 , 5 3 0}\end{array}$ & $\begin{array}{l}\text { Mean follow-up years } \\
\text { (SD) }\end{array}$ \\
\hline O only & 1.00 (ref) & 1.00 (ref) & $2.5(1.4)$ \\
\hline $\mathrm{O}+\mathrm{B}$ & $\begin{array}{l}1.7 \\
(1.62-1.87,<0.0001)\end{array}$ & $\begin{array}{l}1.2 \\
(1.16-1.34,<0.0001)\end{array}$ & $3.0(1.4)$ \\
\hline $\mathrm{O}+\mathrm{S}$ & $\begin{array}{l}1.5 \\
(1.26-1.86,<0.0001)\end{array}$ & $\begin{array}{l}1.2 \\
(1.01-1.50,0.0393)\end{array}$ & $2.9(1.3)$ \\
\hline $\mathrm{O}+\mathrm{B}+\mathrm{S}$ & $\begin{array}{l}2.6 \\
(2.14-3.11,<0.0001)\end{array}$ & $\begin{array}{l}1.6 \\
(1.32-1.93,<0.0001)\end{array}$ & $3.3(1.3)$ \\
\hline
\end{tabular}

$O=$ opioid, $B=$ benzodiazepine, $S=$ non-benzodiazepine sedative-hypnotic

Covariates: gender, region, race (Black, Hispanic, Asian, other), age, Medicaid, depression, anxiety, other psychiatric disorders, substance use disorder, alcoholism, nicotine dependence, previous overdose, sleep apnea, SKM, GABA analogues, buprenorphine, obesity, CCI (weighted) 
Patients aged $\geq 65$ years had nearly 3 times the risk of opioidrelated overdose than those who were between 18 and 44 years of age. Obese patients were $10 \%$ less likely to overdose than nonobese patients. Previous use of buprenorphine or buprenorphine/ naloxone was also found to increase the risk of opioid-related overdose by 2.5 -fold. In addition, those diagnosed with substance use disorder at baseline were associated with approximately 4 times the risk of opioid-related overdose. However, the highest risk was seen in those with a history of previous overdose at 6.7 times the risk of those without a history of previous overdose (Figure 3).

\section{DISCUSSION}

Overall, our results validated that within the commercially insured population, there is a significant increase in the risk of opioid-related overdose in patients exposed to combinations of sedative-hypnotics with opioids compared to those only exposed to opioids, with the highest risk seen in those exposed to opioids, benzodiazepines, and non-benzodiazepine sedative-hypnotics at opioid doses $>20 \mathrm{MME} /$ day.

In addition, we observed an increased risk of overdose with increasing opioid dose in all opioid users, with or without sedative-hypnotics. These findings are consistent with what has been reported in previous literature ${ }^{6,11-13}$.

Results from sensitivity analysis no. 1 did not change previous findings regarding the outcome but revealed that over half of opioid overdose events occurred in "remote users." This may indicate that a large volume of patients had stockpiled opioids from previous prescriptions or obtained opioids outside our integrated system, potentially illegally.

Factors associated with risk of opioid-related overdose were also examined. Results showed that Hispanic race and Asian race as well as obesity were associated with a statistically significant lower risk of opioid-related overdose. However, a recent study conducted in a commercially insured population demonstrated a $26 \%$ higher risk of overdose in obese patients ${ }^{14}$. Upon further investigation, we found that obese patients in our study were less likely to be in the exposure group with the highest risk of overdose, $\mathrm{O}+\mathrm{B}+\mathrm{S}$, comprising only $32 \%$ of this group. This may explain why obese patients within our cohort had a slightly lower risk of opioid-related overdose than those who were not obese. Regarding race as a risk factor, our study validates findings from previous literature and data that demonstrate that White persons are at a higher risk for opioid-related outcomes, such as respiratory depression, overdose or death ${ }^{14,15}$.

The strongest risk factors for opioid-related overdose (aHR $>2.0$ ) were age $\geq 65$ years, previous use of buprenorphine or buprenorphine/naloxone, substance use disorder, and history of previous overdose.

At first glance, it appears that previous use of buprenorphine is linked with an over 2-fold higher risk of opioid-related overdose. In our analysis, we specifically looked at the use of buprenorphine or buprenorphine/ naloxone for substance use disorder indications. We suspected that the increased risk of overdose in these patients may have been because they were more likely to have had a previous overdose or substance use disorder, which are well-known risk factors for opioid overdose ${ }^{14}$. In investigating further, we found that this group had a higher percentage of previous overdose than those without previous use of buprenorphine or buprenorphine/naloxone (3.3\% vs. $0.06 \%$ ). In addition, the majority $(89.3 \%)$ of those who had the outcome and previous use of buprenorphine or buprenorphine/naloxone had a history of substance use disorder. Because previous use of buprenorphine or buprenorphine/naloxone is highly correlated with substance use disorder, we cannot definitively conclude

Table 3 Risk of Overdose Based on Opioid Dosage Strength

\begin{tabular}{|c|c|c|c|}
\hline Exposure groups & $n(\%)$ & $\begin{array}{l}\text { Unadjusted HR } \\
(95 \% C I, p \text { value) } \\
N=\mathbf{2 , 2 4 1 , 5 3 0}\end{array}$ & $\begin{array}{l}\text { Adjusted HR* } \\
\text { (95\% CI, } p \text { value) } \\
N=\mathbf{2 , 2 4 1 , 5 3 0}\end{array}$ \\
\hline $\begin{array}{l}\text { O only, MME } \\
\cdot \leq 20 \\
\cdot 21-49 \\
\cdot 50-89 \\
\cdot \geq 90 \\
\mathrm{O}+\mathrm{B}, \mathrm{MME} \\
\cdot \leq 20 \\
\cdot>20 \\
\mathrm{O}+\mathrm{S}, \mathrm{MME} \\
\cdot \leq 20 \\
\cdot>20 \\
\mathrm{O}+\mathrm{B}+\mathrm{S}, \mathrm{MME} \\
\cdot \leq 20 \\
\cdot>20\end{array}$ & $\begin{array}{l}1,882,478(84.0) \\
29,807(1.3) \\
1,681(0.07) \\
264(0.01) \\
268,494(12.0) \\
7,950(0.35) \\
\\
30,686(1.4) \\
1,222(0.05) \\
17,982(0.8) \\
966(0.04)\end{array}$ & $\begin{array}{l}1.0 \text { (ref) } \\
3.9(3.4-4.5,<0.0001) \\
7.2(4.7-11.2,<0.0001) \\
10.8(4.5-26.0,<0.0001) \\
1.7(1.6-1.8,<0.0001) \\
6.9(5.7-8.3,<0.0001) \\
1.5(1.2-1.9,<0.0001) \\
3.8(1.0-7.2,<0.0001) \\
2.3(1.9-2.8,<0.0001) \\
10.7(7.1-16.1,<0.0001)\end{array}$ & $\begin{array}{l}1.0(\text { ref }) \\
2.7(2.3-3.1,<0.0001) \\
3.8(2.4-5.8,<0.0001) \\
3.1(1.3-7.6,0.007) \\
1.2(1.1-1.3,<0.0001) \\
3.1(2.5-3.7,<0.0001) \\
1.3(1.0-1.5,0.03) \\
2.3(1.2-4.5,0.01) \\
1.5(1.2-1.8,0.0003) \\
4.2(2.8-6.3,<0.0001)\end{array}$ \\
\hline
\end{tabular}

$O=$ opioid, $B=$ benzodiazepine, $S=$ non-benzodiazepine sedative-hypnotic, $M M E=$ morphine milligram equivalents

*Covariates: gender, region, race (Black, Hispanic, Asian, other), age, Medicaid, depression, anxiety, other psychiatric disorders, substance use disorder, alcoholism, nicotine dependence, previous overdose, sleep apnea, SKM, GABA analogues, buprenorphine, obesity, CCI (weighted) 
that previous use of buprenorphine or buprenorphine/ naloxone increases the risk of opioid-related overdose. This increased risk seen with buprenorphine use may have been observed due to association bias and thus we cannot consider previous use of buprenorphine as an independent risk factor for opioid overdose.

Moderately associated risk factors $(\mathrm{aHR}=1.1-2.0)$ included female sex, Medicaid status, depression, anxiety, alcoholism, nicotine dependence, sleep apnea, use of SMRs, use of GABA-analogues, and weighted baseline CCI.

The strengths of this study include the large sample size and the ability to use KP's integrated data system to analyze detailed prescription data and perform a real-world analysis of opioidrelated overdoses. We also considered and adjusted for multiple variables with significant clinical impact. Additionally, we captured both internal and external data through claims.

There were several limitations identified in this study. First and foremost, this was a retrospective study which may introduce selection bias. Because we required an opioid-free interval before the index prescription date, this may limit the generalizability of our results to all opioid users. However, only $10.8 \%$ of our cohort were excluded due to this criterion. Secondly, no chart reviews were conducted on overdose events so we relied on the accuracy of the diagnostic codes used. Because we used pharmacy utilization data, we only know the prescribed dose and not the actual dose of opioids ingested. Another limitation of this study is that we did not assess the risk of overdose based on the type of opioid or duration of opioid use. In the main analysis, we looked at overall exposure, not just at the time at overdose, in order to account for patient behaviors and the possibility of stockpiling. However, we conducted an additional analysis to assess crude rates based on exposure at the time of overdose that confirmed findings from the main analysis. In addition, the rate of opioid overdoses identified in this study may be an underestimation as fatal opioid overdoses that may have occurred outside of the hospital system were not captured.

In conclusion, we observed an increase in opioid overdose risk with increasing opioid dose across all four exposure groups. This demonstrates that opioids should be used at the lowest possible dose. We identified that those who were 65 years and older had nearly 3 times the overdose risk of those aged $18-44$ years. This finding provides evidence that opioids should be used cautiously in the elderly and avoided if possible. This study also demonstrates that those exposed to all three medication classes, opioids, benzodiazepines, and nonbenzodiazepine sedative-hypnotics, had a $60 \%$ higher risk of overdose and a higher percentage of depression and anxiety at baseline than those exposed to opioids alone. Thus, we can conclude that use of opioids with benzodiazepines and/or non- benzodiazepine sedative-hypnotics should be avoided. If exposure to both opioids and sedative-hypnotics cannot be avoided, keeping the opioid dose $<20 \mathrm{MME} /$ day is crucial to keep the risk of opioid-related overdose as low as possible.

Further studies are recommended to assess other factors that may be associated with overdose, such as physician specialty and the impact of opioid dose tapering and discontinuation efforts on opioid overdose risk.

Acknowledgments: Contributors: The authors would like to thank Carol Havens, MD, Doris J. Kao, PharmD, BCPS, FCSHP, Jane Takagi, PharmD, FCSHP, FASHP, Maisha Draves, MD, and Sameer Awsare, MD for their support.

Corresponding Author: Joanne Cho, PharmD, BCPS; Pharmacy Services Kaiser Permanente, Bakersfield, CA, USA (e-mail: joanne.s. cho@kp.org).

\section{Compliance with Ethical Standards:}

Conflict of Interest: Dr. Steven G. Steinberg is currently employed by Southern California Permanente Medical Group. All remaining authors declare that they do not have a conflict of interest.

\section{REFERENCES}

1. Dowell D, Haegerich T, and Chou R. CDC guideline for prescribing opioids for chronic pain-United States, 2016. JAMA. 315.15 (2016): 1624-1645.

2. Substance Abuse and Mental Health Services Administration, Center for Behavioral Health Statistics and Quality.The DAWN Report: Highlights of the 2011 Drug Abuse Warning Network (DAWN) Findings on DrugRelated Emergency Department Visits. February 22, 2013. Available at https://www.samhsa.gov/data/sites/default/files /DAWN 127 / DAWN127/sr127-DAWN-highlights.htm. Accessed August 27, 2019.

3. Benzodiazepines and Opioids. National Institute on Drug Abuse. https:// www.drugabuse.gov/drugs-abuse/opioids/benzodiazepines-opioids. Last updated March 2018. Accessed August 27, 2019.

4. Sun E, Dixit A, Humphreys K, et al. Association between concurrent use of prescription opioids and benzodiazepines and overdose: retrospective analysis. BMJ. 356 (2017): j760.

5. Park T, Saitz R, Ganoczy D, et al. Benzodiazepine prescribing patterns and deaths from drug overdose among US veterans receiving opioid analgesics: case-cohort study. BMJ. 350 (2015): h2698.

6. Garg R, Fulton-Kehoe D, and Franklin G. Patterns of Opioid Use and Risk of Opioid Overdose Death Among Medicaid Patients. Medical Care 55.7 (2017): 661-668.

7. Kaiser Permanente. Fast Facts About Kaiser Permanente. 2018. Available at https://about.kaiserpermanente.org/who-we-are/fast-facts. Accessed August 27, 2019.

8. Losby J, Hyatt J, Kanter M, et al. Safer and more appropriate opioid prescribing: a large healthcare system's comprehensive approach. J Eval in Clin Practice (2017).

9. Prescription Drug Monitoring Program Training and Technical Assistance Center at Brandeis University. Technical assistance guide No. 01-13: Calculating daily morphine milligram equivalents. 2013. Available at: http://www.pdmpassist.org/pdf/BJA_performance_measure_aid_MME_ conversion.pdf. Accessed August 27, 2019.

10. Quan H, Sundararajan V, Halfon $\mathbf{P}$, et al. Coding algorithms for defining comorbidities in ICD-9-CM and ICD-10 administrative data. Med Care. 2005; 43(11):1130-1139

11. Bohnert AS, Valenstein M, Bair MJ, et al. Association between opioid prescribing patterns and opioid overdose-related deaths. JAMA. 2011;305(13):1315-1321. 
12. Dunn KM, Saunders KW, Rutter CM, et al. Opioid prescriptions for chronic pain and overdose: a cohort study. Ann Intern Med. 2010; 152(2):85-92.

13. Gomes T, Mamdani MM, Dhalla IA, et al. Opioid dose and drug-related mortality in patients with nonmalignant pain. Arch Intern Med. 2011;171:686-691.

14. Nadpara PA, Joyce AR, Murrelle EL, et al. Risk Factors for Serious Prescription Opioid-Induced Respiratory Depression or Overdose: Com- parison of Commercially Insured and Veterans Health Affairs Populations. Pain Medicine. 2017;19(1):79-96.

15. Henry $\mathbf{J}$ Kaiser Family Foundation. Opioid Overdose Deaths by Race/Ethnicity. Available at https://www.kff.org/other/state-indicator/ opioid-overdose-deaths-by-raceethnicity/. Accessed August 27, 2019.

Publisher's Note Springer Nature remains neutral with regard to jurisdictional claims in published maps and institutional affiliations. 